\title{
UNA APROXIMACIÓN ANALÍTICA AL CONCEPTO DE FEMICIDIO
}

\section{AN ANALYTICAL APPROACH TO THE CONCEPT OF FEMICIDE}

\author{
Christian EsCOBAR-JimÉNEZ \\ Pontificia Universidad Católica del Ecuador*
}

Resumen: Este artículo propone una aproximación crítica al concepto de femicidio. Se repasa su origen y las diferentes versiones desarrolladas por su proponente, Diana Russell, a más de algunas otras alternativas de la definición. Se realiza una aproximación analítica al mismo, y se revisan sus cualidades intensionales y connotativas. Se hace una breve relación con respecto al concepto alternativo de "feminicidio" y se desarrolla una crítica a ambos. Se refuta la idea de femicidio como un delito de odio y se termina con un análisis sobre las dificultades de la instrumentalización jurídica del concepto, tal como ha sido propuesto.

Palabras clave: Femicidio, feminicidio, delito de odio, filosofía analítica.

AвSTRACT: This paper proposes a critical approach to the concept of femicide from its origin to the different versions developed by its proponent, Diana Russell. I take an analytical approach - in the philosophical sense - to the concept, to understand its intensional and denotative qualities. I also analyse the alternative definition of feminicidio. The idea of femicide as a kind of hate crime is refuted. The papers finishes with an analysis of the difficulties for a legal instrumentalization of this concept as it has been proposed.

KeYwords: Femicide, feminicidio, femicide as hate crime, analytical approach to femicide

* Profesor de la Escuela de Sociología y Ciencias Políticas. Quito - Ecuador. Av. 12 de octubre 1076 y Roca. cmescobar@puce.edu.ec, cmescogen@hotmail.es 


\section{Introducción}

El objetivo de este artículo es debatir la consistencia lógica del concepto de femicidio propuesto por primera vez por Diana Russell, así como analizar algunas otras variantes. En ese sentido, este trabajo es un análisis de segundo orden, es decir, trata sobre un concepto y no directamente sobre la problemática -la violencia extrema de género- del cual el término pretende dar cuenta. Se debate el concepto no como un mero ejercicio semántico o lógico, sino porque éste ha sido adoptado en la tipificación de varios códigos penales en América Latina ${ }^{1}$; por tanto, de su mayor o menor consistencia también se sigue su aplicabilidad.

En 1976, la socióloga norteamericana Diana Russell ${ }^{2}$ usó y reivindicó, por primera vez, el término femicide ${ }^{3}$, para aludir a la violencia sistemática contra las mujeres que termina en muerte. En los primeros días de marzo de aquel año, se organizó en Bruselas un encuentro sobre la violencia extrema contra las mujeres, llamado International Tribunal on Crimes Against Women (Tribunal Internacional de crímenes contra las mujeres). Este encuentro buscaba ser un tribunal popular ${ }^{4}$ para denunciar la violencia contra las mujeres como un conjunto de actos sistemáticos, propios de una estructura social que los legitima, y no como casos aislados de violencia extrema.

El término que Russell puso en juego estaba más vinculado a las corrientes feministas agrupadas bajo el título de Continental Feminism, centradas más en el uso de herramientas del psicoanálisis, crítica posmoderna, fenomenología o en

\footnotetext{
${ }^{1}$ Para mayor referencia, ver el trabajo de Thiago Pierobom de Ávila (2018) The Criminalisation of Femicide. Según el autor, para el año 2018, dieciséis países de América Latina habían adoptado alguna forma de tipificación.

${ }^{2}$ La profesora Russell falleció en julio de 2020, durante el dictamen de este trabajo. Entendemos que cualquier ejercicio crítico es también una forma de dar cuenta de la relevancia en diferentes ámbitos que ha tenido du concepto y, en este sentido, es también una manera de homenaje.

${ }^{3}$ De acuerdo a Silvia Chejter (2008), Russell lo atribuiría, a su vez, a la socióloga norteamericana Carol Orlock, de quien lo había escuchado en 1974 (la propia Russell dice que se lo refirieron en 1975, pues Orlock habría estado preparando un trabajo sobre este tema que no se publicaría). El término le habría parecido perfectamente adecuado para evidenciar el problema de la violencia estructural contra la mujer que termina en la muerte.

${ }^{4} \mathrm{La}$ tradición de tribunal popular puede remontarse al provocatio ad populum de la república romana y terminar en los Volksgerichtshof nazis, por lo que su uso es bastante disímil (Humbert, 1988). En el caso del Tribunal Internacional, tenía un objetivo más bien exhortativo que de juzgamiento y perseguía la discusión teórica.
} 
el análisis y deconstrucción de acciones y discursos de legitimación de la violencia de género y denuncia de los factores ideológicos que están detrás (Hansen, 2019). En los años previos al Tribunal antes mencionado, todavía no existía una distinción clara entre las diferentes "olas" o "tipos" de corrientes feministas que escritoras como Alison Jaggar ayudaron a consolidar, para tener una mejor perspectiva de análisis y entender la evolución de las ideas de un cuerpo común de conocimiento. Este cuerpo común puede entenderse como un compromiso intelectual y político que busca la justicia para las mujeres, así como el fin de la opresión por razones de género en todas sus formas (McAffe, 2018).

$\mathrm{Al}$ parecer, ya en 1801 el término femicide habría sido usado en un diario londinense para referirse al asesinato de una mujer (Chejter, 2008). Otra versión (Russell, 2009; Toledo Vásquez, 2009) es que el término aparece en el mismo año en el libro de John Corry, titulado A Satirical View of London at the Commencement of the Nineteenth Century. En este libro se daba una perspectiva general y crítica sobre la composición demográfica, económica y social de Londres en los tiempos convulsos de la revolución industrial.

Este primer uso del término, introducido por Corry, se entiende como una alternativa a la palabra "homicidio" y sigue la misma línea de la definición del tipo de asesinato por el objeto o la víctima, tal como sucede en palabras como uxoricidio, parricidio, deicidio, infanticidio, filicidio, etc. Por supuesto, la intención de Russell iba más allá de este uso y evidenciaba todo un trabajo teórico y político inexistente a inicios del siglo XIX. "One of the most important tasks when talking about femicide is to clarify its difference from woman homicide. While Female Homicide is any murder of women or girls regardless of the circumstances, Femicide is the murder of women or girls for gender-based reasons" (Saccomano, 2015: 4).

Así, la introducción de esta palabra en los estudios de género denuncia o desenmascara una forma de violencia extrema que obedece a las estructuras de dominación de un género sobre otro. De esta forma, Russell define por primera vez al femicidio como "el asesinato de una mujer por parte de un hombre por el hecho de ser mujer" (Russell, 2009).

Caputi y Russell (1990) conceptualizan al femicidio como la muerte de mujeres por parte de hombres "motivados por el desprecio, el odio, el placer o el sentimiento de propiedad...”. El femicidio es una forma extrema de violencia 
que se sigue de prácticas patriarcales constantes a lo largo de la historia de la humanidad:

We must realize that a lot of homicide is in fact femicide. We must recognize the sexual politics of murder. From the burning of witches in the past, to the more recent widespread custom of female infanticide in many societies, to the killing of women for «honor,» we realize that femicide has been going on a long time. But since it involves mere females, there was no name for it before the term femicide was coined. (Russell, 2012: 1).

Con esta palabra, Russell buscaba constatar dos cosas. La primera es que existe toda una trayectoria histórica y estructural de violencia contra la mujer, desde los mitos fundacionales de las religiones monoteístas, la caza de brujas en la Edad Media o en los Estados Unidos del siglo XVI, hasta espeluznantes formas contemporáneas de violencia como el snuff, en el que la mayoría de las víctimas son mujeres. Además, estas estructuras de violencia cobran un cariz particular de acuerdo a cada cultura: la dilapidación a mujeres acusadas de infidelidad entre los chiitas; ablaciones genitales, clitoridectomías u otras formas de mutilaciones genitales a mujeres; asesinatos por honor o los llamados asesinatos pasionales, etc.

La segunda es que tal condición es generalizada y no particularizada, por tanto, es estructural (Marín de Espinosa, 2017); es decir, que las víctimas lo son por el hecho de ser mujeres, por su condición de género, lo cual conecta al femicidio con crímenes por condiciones esenciales adjudicadas a las víctimas, como asesinatos por motivos xenófobos, racistas, antisemitas, religiosos, etc. La idea sería que "no son hechos aislados atribuibles a factores puramente individuales, sino que responden a casusas estructurales, a la sumisión en que la sociedad patriarcal sitúa a las mujeres como colectivo subordinado" (Laurenzo Capello, 2012: 121). Por tanto, "por el hecho de ser mujer".

Russell afirmaba que, para 1992, en el libro escrito con Jill Radford, el término femicidio varío o se redujo a "the misogynist killing of women by men", para volver al 2001 a ser "the killing of females by males because they are female", pero dado que las mujeres pueden ser víctimas de actos machistas cometidos por

\footnotetext{
${ }^{5}$ El snuff es una forma extrema de pornografía, en la que se graba el asesinato de personas, generalmente mujeres, a las que se somete previamente a todo tipo de vejámenes - torturas, descuartizamientos, violación, etc. Al parecer, resulta difícil establecer si los videos que circulan son reales o montajes.
} 
mujeres, en el 2012 lo cambia a "femicide is the killing of a female because she is a female". De acuerdo con la propia Russell, la definición más completa podría ser la que ella dio junto a Roberta Harmes en 2001: "el asesinato de personas del sexo femenino por personas del sexo masculino debido a su condición de ser personas del sexo femenino" (Russell, 2009: 42; Russell y Harmes, 2001). La autora recalca la sustitución de "masculino" por "hombres" y "femenino" por "mujeres", para extender el universo de los victimarios y de las víctimas a niños y niñas, respectivamente (o adolescentes).

En resumen, tendríamos las siguientes posibilidades no exhaustivas de definición:

1. Un asesinato cuando la víctima es una mujer - John Corry.

2. El asesinato de una mujer por el hecho de ser mujer - Diane Russell.

3. El asesinato de una mujer por parte de un hombre por el hecho de ser mujer.

4. El asesinato de una mujer por motivos misóginos.

5. El asesinato de personas del sexo femenino por parte de personas del sexo masculino por el hecho de pertenecer al sexo femenino.

Todas las definiciones, excepto la primera, tienen presente una connotación política, es decir, el propio concepto denuncia y connota un tipo de condiciones entendidas como estructurales en las relaciones de género. La segunda definición añade una motivación, mientras la tercera, a más de la motivación de la segunda, suma el sexo o género del victimario como condición. Además, la tercera aúna como motivación la misoginia, lo que puede vincularla a la definición más estándar de Russell. Por último, en la quinta, se sigue en el patrón de la segunda, pero se amplía el universo por una consideración etaria.

Por otro lado, quisiera recalcar que el concepto de Russell lleva implícito una concepción binaria en el que dos conjuntos cuyos elementos no son intercambiables agotan todas las posibilidades. En el concepto se comprenden las condiciones de víctima y victimario en las que no hay la posibilidad de un tercero, sencillamente, porque connotativamente se atiene a la condición biológica de los miembros de cada grupo. Esta aclaración no tiene por objeto 
acusar el binarismo de la concepción en el sentido en el que se suele criticar desde el feminismo, sino sumar un elemento para el análisis. En todo caso, la idea de una mujer (en este caso, usaremos "mujer" y "sexo femenino" como términos intercambiables) asesinada por un hombre por el hecho de serlo, se contextualiza (ya sea biológicamente o por tradición cultural), como los dos únicos subconjuntos del conjunto humanidad (para el concepto es irrelevante el problema de la autoidentificación de género, intersexualidad, etc.) ${ }^{6}$.

\section{Femicidio}

Como se ha dicho, la acepción de femicidio desarrollada por Russell no solo denota el sujeto pasivo asesinado, es decir, en quien recae la acción ${ }^{7}$ : una mujer; por lo cual es similar a los casos de parricidio, deicidio, etc.; sobre todo, el concepto pone énfasis en el complemento de la proposición, no únicamente en el predicado: "es el asesinato de una mujer por el hecho de serlo". Su función connotativa intenta revelar una condición de violencia estructural y socialmente legítima contra las mujeres. Por tanto, el punto clave es tanto el objeto pasivo como la motivación, que se convierte en condición necesaria del concepto.

Este concepto presenta complicaciones lógicas en los siguientes sentidos:

1. La definición comprende un conjunto universo compuesto por solo dos subconjuntos: hombres $(H)$ y mujeres $(M)$. Dichos conjuntos no tienen intersección posible, por lo que los elementos de cada uno son disyuntivos y no pueden pertenecer a uno y otro a la vez. Por tanto, cada elemento pertenece a un conjunto y solo a uno de los dos posibles, dado cada uno por la propiedad de ser hombre o ser mujer (digamos que el criterio es biológico o de autodefinición, da igual). La intensión de $M$ o $H$ es mutuamente excluyente. $H$ y $M$ se definen en la medida en el que sus elementos extensionales pertenecen a un conjunto y solo a uno. Todo $h$ pertenece a $H$ y solo a $H$ y cada $m$ pertenece a $M$ y solo a $M$. Entonces, sería imposible que un $h$ mate a un $m$ que no pertenezca a $M$, es decir, que no sea

\footnotetext{
${ }^{6}$ En un primer momento, es irrelevante la autodefinición de género y la condición intersex, ya sea porque se adscribe a la denotación cultural de relación binaria o por los motivos de estructuración de poder misógino, que convierte a las mujeres en objeto de odio de los hombres.

${ }^{7}$ En el sentido de objeto directo de los verbos transitivos "asesinar", "matar", etc.
} 
elemento del conjunto $M$. Así, es lógicamente imposible matar a un $m$ por el hecho de no serlo. A esta crítica se le podría objetar que es una obviedad que una persona no mata a otra por el "hecho de no serlo" (excepto quizá en un homicidio accidental no culposo) y que la intención de Russell es la de contextualizar los crímenes como un problema estructural y no comprenderlos como casos aislados o etiológicamente clasificados. Sin embargo, esto no elimina el problema lógico-estructural de una proposición que se ha incorporado a diversas legislaciones en el mundo y que puede tener problemas de instrumentalización jurídica.

2. Se puede hacer una crítica similar desde la comprensión de los juicios analíticos y su relación con las tautologías. ${ }^{8}$ En su famosa distinción entre juicios analíticos y sintéticos, Kant $(1999 ; 2002)$ nos dice que en los juicios analíticos hay un enlace entre sujeto y predicado, en términos de identidad". El ejemplo clásico es "todos los cuerpos son extensos", la propia definición de cuerpo implica extensión y, por tanto, se opone a la nada (no extensa). La extensión es parte de la identidad de cuerpo. La relación nada y algo es excluyente, algo similar al tercero excluido. $O$ se es o no se es, no hay otra opción. Al igual que en la definición de Russell, o se es hombre o se es mujer, no existe otra posibilidad. Ahora bien, en lógica, el principio de identidad es una propiedad por la cual todo objeto es idéntico a sí mismo, de ahí se derivan leyes de identidad, entre las cuales se hallan las tautologías. Así, todo objeto es igual a sí mismo. La idea subyacente en las reglas lógicas desde Aristóteles es que al ser idéntico a sí mismo tampoco pueda ser diferente de $s^{10}{ }^{10}$, por tanto, nada puede ser ni lo que no es ni dejar de ser lo que es. De esta manera, el complemento "por el hecho de ser mujer" se torna tautológico o turbio, porque una mujer no podría dejar de ser mujer - al igual que todo lo demás. Por ello, como proposición, el complemento se vuelve innecesario, aunque irónicamente es una condición necesaria de la definición.

\footnotetext{
8 "El término identitas habría sido formado, en el bajo latín, para traducir al

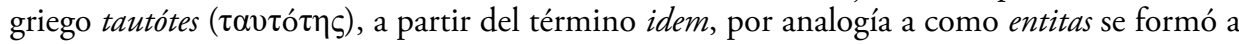
partir del término ens" (Bueno, 1999: 3).

9 En filosofía, la distinción entre los juicios analíticos y sintéticos ha sido ampliamente debatida, quizá la refutación más famosa sea la de Quine. La distinción se ha descontinuado, pero sirve para la discusión aquí expuesta.

${ }_{10}$ Lo que tiene repercusiones metafísicas al idealizar objetos estables en el tiempo, presupuesta una substancia o ousía.
} 
3. Siguiendo la argumentación anterior, si se introduce el complemento "por el hecho de ser mujer", entonces, existen asesinatos posibles en los que la víctima es una mujer, pero no ha sido asesinada por "el hecho de serlo"; por tanto, se debe determinar esta diferencia, ¿cuándo alguien mata a alguien por el hecho de serlo o no serlo? (como el ejemplo dado de las muertes accidentales). También se puede voltear la pregunta, ¿̇más allá de las muertes accidentales, existe la posibilidad de matar a una persona que "no lo sea"? o aún ¿más allá de las muertes accidentales, existe la posibilidad de matar a alguien por el hecho de no serlo?

4. De forma abstracta es más difícil comprender las limitaciones del concepto, pero las siguientes preguntas evidencian el carácter problemático de esta definición: ¿¿cuándo un hombre mata a una mujer a "pesar de serlo"? ¿si una mujer mata a otra "por el hecho de serlo", se considera femicidio? y ya en términos jurídicos ¿̇i una persona es víctima de otra por el hecho de serlo o no serlo, cómo podemos hacer tal diferencia y, sobre todo, demostrarlo?

Se entiende que el concepto tiene sentido en la connotación de un determinado tipo de estructuras sociales que vuelven a la mujer vulnerable y frecuentemente víctima de asesinatos; sin embargo, en el momento de tener una proposición que pueda instrumentalizarse jurídicamente, esto se convierte en un fárrago.

Por último, y como se anticipó, cuando Russell enlaza el vínculo victimariohombre y víctima-mujer, establece una relación binaria. Evidentemente, al insertarse en la tradición del feminismo continental, este binarismo no es "aceptado" por Russell como un "hecho" biológico, unívoco y real, sino que es concebido instrumentalmente para su definición, ya sea para connotar los problemas estructurales de la violencia de género o para evidenciar la concepción binaria de la sociedad. En todo caso, la definición debe aclararse cuando entramos en el terreno de la autodefinición de género. La definición no parte de una "esencialización" del sexo y debería convivir con algo similar a la idea de "performatividad" del sexo en el sentido de Butler (2007), siguiendo las propias líneas teóricas en las que la definición de femicidio se enmarca.

Este problema se puede traducir en las siguientes preguntas: ¡es necesario que una "mujer biológica" se "autodefina como hombre" para que sea victimaria/o? $\mathrm{O}$ a la inversa ¿es necesario que un "hombre biológico" se considere "mujer" para poder ser víctima y tipificarse como femicidio? ¿Cómo se compadece el 
concepto de Russell con las teorías de la performatividad del género? ¿Si un hombre biológico mata a una mujer por el hecho de serlo, puede argumentar como descargo que se autoidentifica como mujer?

\section{3. ¿Por qué no funciona de la misma forma que en los delitos de odio?}

Los delitos de odio pueden ser definidos de la siguiente manera: "hate crime is a criminal act motivated, at least in part, by the victim's group. Hate crime is sometimes also called by other names such as 'bias crime' or 'ethnic intimidation"” (Gerstenfeld, 2017: 1). Como derechos, aparecieron después de la Guerra Civil americana, sobre todo, para proteger a los antiguos esclavos del Sur de las crecientes agresiones impersonales que los afrodescendientes sufrían por su condición étnica o racial. Existen tres aspectos relevantes en este tipo de delitos: "la presencia de una aversión discriminatoria, las necesidades preventivas de los colectivos vulnerables y la defensa de los valores de la comunidad puestos en cuestión" (Fuentes Osorio, 2017: 1). Así, para que exista un delito de odio, una persona debe ser víctima de un acto lesivo por el hecho de pertenecer a un grupo o colectivo. El delito de femicidio se evalúa a partir de la pertenencia a un grupo: "por el hecho de ser mujer", como en el caso de delitos racistas, religiosos, xenófobos, etc.

Ahora bien, a modo de contraejemplo a esta idea, expongo los siguientes datos. De acuerdo a un famoso artículo de Campbell et al. (2003), el femicidio es la principal causa de muerte de mujeres jóvenes afroamericanas entre los 15 y 45 años, y la séptima causa de muerte prematura de mujeres en Estados Unidos. En estos casos, los asesinatos cometidos por parejas oscilarían entre el 40 y 50\% del total, por lo que uno de cada dos femicidios es perpetrado por las parejas de las víctimas. Asimismo, para Campbell, Webster y Glass (2009), en los primeros años del siglo XXI, la proporción de muertes de mujeres perpetradas por sus parejas fue 4 veces superior a la muerte de hombres.

Según el Atlas de género (Valle, 2018) del Instituto Nacional de Estadísticas y Censos (INEC) de Ecuador, la violencia de género es mayoritariamente perpetrada por parejas. Carcedo y Ordóñez (2011) señalan que, en Ecuador, entre los años 2005 y 2007, el 66\% de los femicidios analizados fueron cometidos por parejas o ex-parejas. En República Dominicana, entre los ańos 2000 y 2006, el $68 \%$ de los femicidios eran cometidos por las parejas y en el $91 \%$ de los casos, 
los asesinos conocían a sus víctimas (Pola, 2009). De acuerdo con Matthews et al. (2009), cada 6 horas se produce un asesinato íntimo en Sudáfrica - en el que el asesino es la pareja. En México, entre el sexenio 2012-2018, de más de 12.000 asesinatos de mujeres, el 22\% fue investigado como femicidio y el $70 \%$ cometidos en público (Mendoza, 2018). En Argentina, en la primera década del siglo, entre el 50 y $60 \%$ de los femicidios se cometieron por parejas o exparejas (Chejter, 2008). Como se puede constatar por estos datos, en este tema grave y complejo, en la mayoría de los casos, el victimario conoce a su víctima, o incluso se trata de parejas o ex -parejas.

Siguiendo la definición, los delitos de odio suponen una suerte de esencialización imputada desde los victimarios a las víctimas; es decir, la adjudicación de una serie de características que van más allá de su condición individual y que, más bien, las anclan a un grupo. Esta naturalización supone un criterio fundamental para tipificar los delitos de odio y despersonaliza a la víctima (lo es por pertenecer al grupo, como reza la definición de Gerstenfeld). Así, lo importante es que la identidad de la víctima es la del grupo, por lo que su individualidad desaparece, tanto como su nombre propio, su existencia sustantiva, y es esto lo que le da la condición de bias crime (color de piel, lugar de nacimiento, religión). En este mismo sentido, la condición de ser mujer sería la que articula la identidad de la persona y permite la relación desigual que puede llevar al crimen.

Los problemas lógicos al respecto son los siguientes:

1. Se entiende al femicidio como delito de odio por la intención y el móvil de los perpetradores de asesinar a sus víctimas por "el hecho de ser mujer", lo que supone una suerte de esencialización similar a la de los crímenes raciales, religiosos, etc. En los delitos de odio, la tónica es la despersonalización de las víctimas, pues éstas se convierten en tales por pertenecer al grupo; luego, que el perpetrador conozca o no a su víctima no es relevante para la definición (puede serlo en el proceso, como agravante, por ejemplo), lo importante es que comete el crimen por asociar a la víctima al grupo. Ahora, si se toma en cuenta que, al parecer, en la mayoría de los casos, las víctimas y victimarios son o fueron parejas o mantuvieron algún tipo de relación, la aplicación se torna dudosa, porque, en estos casos, la víctima lo es por su condición individual, lo que no se compadece con la definición de los delitos de odio. Las víctimas lo son en su singularidad.

2. Justamente, el cambio de la concepción de "violencia familiar" o "doméstica" a "violencia de género" sigue los presupuestos de que el problema no es 
etiológico, individual o social, sino que es estructural (Marín de Espinosa, 2017). En efecto, tal cantidad de asesinatos cometidos por hombres contra sus ex -parejas da cuenta de que existe un problema social enorme cuyas causas no tienen cabida en este escrito. Sin embargo, si bien el problema puede ser estructural o social, no tiene las mismas connotaciones de los delitos de odio, justamente por la singularidad ya expuesta.

3. Cuando hablamos de delitos como la xenofobia, racismo, intolerancia religiosa, se debe tener en cuenta que, en estos casos, el universo de grupos posibles supera los dos presupuestos en la definición de femicidio: un hombre que mata a una mujer por el hecho de ser mujer. Por ejemplo, los suprematistas blancos se consideran superiores a cualquier otro tipo de "raza" (hispana, amarilla, negra, etc.), es decir, a todos los demás subconjuntos. De la misma manera, la xenofobia implica la identidad del grupo (país), frente a los demás conjuntos de todo el universo. Atacar a un desconocido por ser un elemento de tal o cual grupo es un aspecto del cual se puede extraer claramente indicios objetivos para determinar móviles. Existen grupos políticos con miembros adscritos, con idearios racistas, xenófobos, es decir, criterios de rastreabilidad, en universos con más de dos conjuntos "en conflicto". En sociedades institucionalmente monogámicas y heterosexuales, no existen más opciones posibles que las dos destacadas. Así, en la definición de monogamia heterosexual, si un hombre mata a su pareja, necesariamente será a una mujer. Esto no permite tener criterios de móviles de pertenencia a un grupo, puesto que no hay otros posibles. Así ¿en los casos de asesinatos de mujeres por sus parejas, es posible verificar que el delito ha sido cometido por el "hecho de ser mujer", por su pertenencia al conjunto?

4. De acuerdo con el juez argentino Raúl Zaffaroni, el femicidio no puede ser considerado como un delito de odio porque no conlleva un metamensaje como anuncio del victimario al colectivo social (Racca, 2015). En palabras de Zaffaroni: "El homicidio por odio se produce contra minorías. La característica que tiene es que no importa el individuo. Hay dos lesiones: una al muerto y otra, por el metamensaje, a toda la colectividad. Y acá en la Argentina nadie sale a la calle a matar una mujer porque es mujer. Es una locura, no existe." (El Clarín, 2015). Los delitos tipificados como femicidio no pretenden ser ejemplificadores contra el colectivo "mujer". ${ }^{11}$

\footnotetext{
${ }^{11} \mathrm{Al}$ respecto, es importante notar que hay campañas contra el femicidio que empiezan con eslóganes como "nos están matando", justamente, pensando los asesinatos como como actos ejemplificadores.
} 
5. Una postura similar es la de Sharon Calderón Gordo (2005: 13), cuando critica la idea de cómo la violencia de género centra la disputa entre hombresvictimarios y mujeres-víctimas sin otras posibilidades en cuestión, que no solo se limitan las relaciones de pareja y que también naturalizan la condición masculina: Todas estas medidas de sensibilización y prevención de nada servirán si damos por supuesto que un hombre es violento con una mujer sólo porque es mujer. Habrá otros motivos, por venganza, por envidia, por celos, \&c., pero nunca por ser mujer, nunca por pertenecer al sexo femenino, porque de ser así, un hombre violento se mostraría como tal frente a cualquier mujer, por la calle, en el trabajo, en el autobús..., pero todos sabemos que esto no es así, todos sabemos que el objetivo de un maltratador es muy concreto, no es cualquier mujer, es una mujer. (Calderón Gordo, 2005: 13)

6. ¿En realidad un hombre que asesina a una mujer lo hace porque la odia por el hecho de ser mujer? Sería más plausible que lo haga por muchos otros motivos personales, algunos de los cuales lo evidencian las propias teorías feministas (sentido de posesión, exclusividad y pertenencia como pareja, sentido de superioridad) o factores psicológicos personales no extensibles al resto de la sociedad, es decir, no fruto de la reproducción de las estructuras. Aquí, una pregunta se abre, ¿en el caso de que tales estructuras existan, no se asume de forma fatalista que todos vivimos subsumidos en unas estructuras de las que no podemos escapar, y, además, tenemos motivos misóginos inconscientes que no comprendemos?

7. Wendy Brown (2000) establece una paradoja en cuanto a los derechos que reconocen una condición "femenina". En esta paradoja, los derechos que se atienen al "hecho de ser mujer" como grupo, se limitan a una identidad ya definida por la subordinación (es decir, "esencializan" a la mujer y potencialmente a los hombres); por otro lado, los derechos que no se atienen a esta especificidad de "mujer" no hacen visibles los problemas subyacentes.

8. En este caso, quizá solo los asesinatos que puedan probarse como misóginos puedan ser considerados como delitos de odio en estricto sentido.

En su célebre libro sobre Auschwitz, Lev Poliakov (1969) cita un discurso de Himmler en el que éste insta a la población a acusar a sus vecinos judíos, a pesar de que sean "buenos vecinos y buenas personas". "Todos tenemos un buen judío", dice Himmler. En esa sociedad en la que se premia la delación, la identidad de los grupos en disputa (los arios contra judíos, gitanos, etc.) debe 
subsumir a todos los "judíos buenos vecinos", porque la particularidad no puede estar por encima del grupo. La pertenencia al grupo es la razón fundamental, no es algo accesorio, casual o "inevitable", en la medida en la que el conjunto de posibles se reduce a dos (en el sentido del concepto). Como crimen de odio, funcionaría en el caso de los abortos selectivos practicados en la India o las fosas comunes con cuerpos exclusivamente de mujeres encontrados en Ciudad Juárez, pero difícilmente en los casos de asesinatos de hombres a sus parejas, lo común en la tipificación de femicidio.

\section{Femicidio y feminicidio}

El término "feminicidio" fue introducido por la autora mexicana Marcela Lagarde (2006), para dar cuenta de que en casos como el mexicano (teniendo a Ciudad Juárez como un ejemplo paradigmático) u otros países de América Latina, la estructura de dominación hacia la mujer, incluso en las formas más violentas como los asesinatos, empieza (y termina, en el peor de los sentidos) en el propio Estado. La violencia social, la desinstitucionalización, las mafias enquistadas en el poder, y otras formas de opresión social promueven la violencia contra la mujer y la impunidad.

En general, cuando se estudia la tipificación penal en América Latina y el avance de los llamados derechos de segunda generación (Marín de Espinosa, 2017), se toma a ambas palabras como intercambiables (Toledo Vásquez, 2009), aunque siempre se recalca sus diferencias. El uso del término "feminicidio" suele ser más restringido, sobre todo al ámbito mexicano (Laurenzo Copello, 2012; Marín de Espinosa, 2017), mientras "femicidio" está más extendido en el resto de Iberoamérica. La idea de femicidio pretendería dar cuenta no solo de la responsabilidad del Estado, sino también de delitos de misoginia (Toledo Vásquez, 2009: 27). Lagarde (2006: 217) parte del concepto de "femicidio" de Russell y Radford, que "ubican los homicidios contra nińas y mujeres como parte de la violencia de género”, sumándole una visión que aúna violencia cultural y de las instituciones estatales.

Feminicidio (instead of femicidios) tends to be employed in Spanish as the one notion that best expresses the violent death of women and girls, because it incorporates the semantic field of connivance of state and public institutions as relevant factors in femicide. Ciudad Juárez and the Mexican case reveal the effects of impunity in a context of a failed state. (Corradi et al. 2015: 10) 
Siguiendo a Corradi et al. (2015), la idea de "feminicidio" agrega un semantic field of connivance, al connotar las condiciones políticas que permiten la impunidad. Pero la distinción no está en ello, sino en el contenido. El concepto primigenio ya añade un campo semántico para su interpretación, pues necesita del conocimiento contextual de las estructuras de dominación para poder interpretarlo.

De acuerdo con Russell (2012), fue la misma Lagarde quien le pidió que la traducción de la palabra femicide al español fuese "feminicidio", tomando en cuenta los factores antes anotados. ${ }^{12}$ La crítica de Russell al término de Lagarde es la siguiente:

But it's not appropriate to consider the redefinition of a term as constituting coining it. Furthermore, a sound definition must separate the phenomenon being defined from the response to it. So, for example, if a man who is a wife batterer finally kills his wife, he is likely to be guilty of femicide. If he is arrested and found guilty of this crime, then by Lagarde's definition, he is no longer guilty of feminicide, because the case wasn't treated with impunity. This demonstrates why Lagarde's revised definition of feminicide must be rejected. In addition, it is not a userfriendly term, since it is difficult to pronounce. It took me years to master its pronunciation. Try it yourselves in the lunch break, and compare it with saying femicide. (Russell, 2012: 3)

A mi juicio (más allá de las consideraciones fonéticas), Russell tiene razón con la observación de que añadir la impunidad o la anuencia estatal como condición necesaria para el concepto no aporta a la definición: ¿ si el asesinato de una mujer por el hecho de serlo es juzgado y sancionado, deja de ser feminicidio? En el marco de esta discusión, la distinción entre ambas palabras solo tendría sentido en términos prácticos. El criterio de impunidad por parte del Estado solo aportaría algo en el caso en el que un crimen deba ser judicializado fuera del territorio nacional, una vez agotadas todas las instancias.

Ahora bien, si se observa detenidamente, el concepto de Diana Russell adolece de problemas similares, como en las preguntas ya expuestas: ¿si una mujer mata a otra por el hecho de ser mujer, es femicidio? Y esta diferencia sí tiene

\footnotetext{
${ }^{12}$ Russell (2012: 3) aclara que la petición se debe a: "the impunity with wich these crimes are typically treated in South America” (en realidad, Lagarde centra su análisis en México, no en América del Sur).
} 
implicaciones importantes en la tipificación penal y la judicialización, pues si se considera al femicidio como un delito de odio, la pena sería mayor.

Otra duda que surge en el concepto de feminicidio es la forma en la que esta idea de "sistematicidad", entendida como una forma de institucionalidad determinada, que no solo permite la impunidad, sino la propia violencia, se compadece con la otra, la del carácter orgánico de la violencia de género como una especie de estructura cultural no necesariamente consciente. En todo caso, ambas pueden coexistir, pero no se sabe específicamente cómo.

\section{Imputabilidad, motivación o móvil}

Como se ha anotado, la definición contempla un grupo victimario (hombres o personas de sexo masculino), un conjunto de víctimas (mujeres o personas de sexo femenino) y un móvil (por el hecho de ser mujer). Dada la definición, las condiciones para la tipificación están dadas. Si todas estas condiciones son tomadas como necesarias y ninguna suficiente, para que se catalogue como femicidio se deben cumplir las tres. Dado que el concepto se incorpora a las legislaciones y crea tipos penales, el problema supera el ámbito puramente teórico y entonces aclarar las connotaciones de tales estructuras sociales se vuelve imperante para que la proposición funcione jurídicamente.

¿Cómo determinar las motivaciones y por ende la imputabilidad de un delito que puede tener una pena más alta que la de un homicidio "común"? En su famosa Teoría pura del derecho, Kelsen (1965) había definido a la imputación como una relación entre acto, responsabilidad y pena. La causalidad jurídica suponía la existencia de un marco normativo que asocie un acto con una responsabilidad y una sanción consecuente. De acuerdo a Mir Puig (1994: 38-39), en la doctrina jurídica, la imputabilidad se basa en 1) la capacidad de comprender lo injusto del hecho, 2) la capacidad de dirigir la acción conforme a ese entendimiento, en el sentido de conocer o querer cometerlo.

Por su parte, Peláez Mejía (2018: 296) sostiene que la causalidad es un nexo entre el acto ejecutado y el resultado ulterior y que la comprobación es un requisito necesario para considerar la responsabilidad de quien comete el acto, aunque no es suficiente pues además se debe demostrar la vinculación jurídica en un juicio; la demostración del dolo, impericia o cualquier tipo de responsabilidad; así como la conciencia de los actos. Se entiende que, en diferentes códigos, los 
procedimientos y condiciones divergen, pero se siguen a partir de este esquema central.

En cualquier caso, la imputación no solo conoce del acto - digamos, un tipo de criterio objetivo -, sino también de la motivación - un criterio subjetivo -. En el caso del concepto de femicidio, podría existir un criterio objetivo, como el asesinato de una persona de sexo femenino por parte de una de sexo masculino, pero debe acompañarse de una motivación - por el hecho de ser mujer o por el hecho de pertenecer al sexo femenino. La condición necesaria para la tipificación se vuelve difícil de determinar.

The criminal act is always a meaningful assertion produced in the external reality. The meaning is only deduced from this production. As an example, if the meaningful claim is taken out of "killing someone", then there is no prohibition to kill. The criminal act as a meaningful claim deals with an activity in the concrete "real" world and thus necessarily deals with the rules of the environment. (Jakob, 2004: 501)

Estas reglas están claramente estipuladas (una sanción proporcional al acto socialmente lesivo de los asesinatos), y su sentido se establece en esa relación, entre la norma y la posibilidad de discernimiento de los actos al amparo de tales normas (lo que Jakob llama "socialmente significante", pues, para él, en un sentido convencional a lo David Lewis, una sociedad existe cuando hay al menos una norma a seguir). Un acto criminal que tiene una relación con una norma debe considerarse dentro de cierta objetividad, pues en la dilucidación de los hechos y su verdad se juega nada menos que la posibilidad de "justicia"13. Cuando se juzga la prueba con cierta objetividad se dispone de un criterio de verdad para llevar el caso y dilucidar lo ocurrido (Tarufo, 2008). Si bien la condición necesaria para la tipificación "por el hecho de ser mujer", en su sentido connotativo, puede tener un contenido valioso, teóricamente hablando, y puede permitir que se evidencien o delaten las estructuras de poder implícitas, en un sentido jurídico, no tiene grandes posibilidades instrumentales y de aplicación objetiva ${ }^{14}$.

\footnotetext{
${ }^{13}$ En el sentido de conocido por la institucionalidad garante del cumplimiento de las normas y susceptible de sanción, para que un acto con consecuencias jurídicas establecidas no quede en la impunidad.

${ }^{14}$ Un ejemplo de esta dificultad es la sostenida por Tuesta y Mujica (2015) para el caso peruano, quienes afirman que en los juicios por femicidios existe una enorme dificultad probatoria cuando se trata de criterios para discernir la discriminación de género y consecuentemente encontrar elementos materiales para sustentar los casos.
} 
En ese sentido, en el caso en el que los elementos materiales probatorios no puedan "demostrarse" de manera tal que se asocien a formas de discriminación de género, solo queda "asumir" previamente que tal discriminación existe por motivos estructurales. Este elemento es clave, pues los actos se juzgan por su imputabilidad a los individuos que los cometen y por las particularidades de los móviles. Apelar a motivos estructurales es ir en contra el debido proceso porque en derecho los actos deben juzgarse por quien los comete y sus móviles particulares, no por el grupo al que pertenece (en este caso, la idea de la violencia de los hombres sobre las mujeres). Si en una sociedad determinada existen elementos para pensar que un grupo atenta contra los derechos de otro, esto no es condición suficiente para asumir que dado que la víctima y el acusado pertenecen a grupos vulnerables y privilegiados, respectivamente, el acusado es culpable por pertenecer a dicho grupo. Esto es justamente lo que sucede en To kill a Mockingbird de Harper Lee, donde el acusado es sentenciado por pertenecer a un grupo: sencillamente es negro.

En efecto, en sentido lato, existen razones de peso bien fundamentadas para pensar que las mujeres o personas que se autoidentifican como tales son más vulnerables que los hombres, sobre todo en lo que tiene que ver con violencia física; basta con ver los datos de quiénes cometen los asesinatos y quiénes son las víctimas. Pero lo que tiene sentido teórico y estadístico no puede ser un elemento procesal en el sentido de que cada imputado es único, aunque no esté sustraido ni de su grupo ni de su contexto social. Probar procesalmente que alguien muere "por el hecho de ser mujer" es extremadamente difícil, por la subjetividad de la condición y porque, como se ha dicho, lógicamente, no puede no serlo.

Por lo general, la idea de violencia de género no solo se enmarca en esta comprensión de un problema estructural, como afirma Marín de Espinosa (2017), sino que se suele asociar únicamente a violencia contra la mujer, justamente por esta condición estructural. Sharon Calderón Gordo (2005) argumenta contra la falta de atinencia de la etiqueta "violencia de género", trasladado desde la academia norteamericana al ámbito hispanoamericano, cuando se usa de forma intercambiable con "violencia contra la mujer". Usarlo así desconocería los otros "géneros" posibles y no atiende a la potencial violencia inversa, basándose únicamente en criterios de generalidad. Para esta autora, esta idea "lejos de conseguir una mayor «sensibilización», no es más que otra muestra del dislate ideológico que arrastra una ley que culpabiliza a los hombres "por el hecho de serlo»" (Calderón Gordo, 2005: 13). 


\section{Algunas conclusiones}

Como afirma Lagarde (2006: 217): “un concepto, una categoría, una mirada, una visión nos permite avanzar muchísimo" y es precisamente en ese sentido en el que se debe repensar el concepto de femicidio. Desde un punto de vista puramente nominal, su valor radica en visibilizar un conjunto de hechos alarmantes, execrables y socialmente lesivos que deben tratarse sin impunidad y con justicia. La pregunta sería ¿en realidad, la condición necesaria "por el hecho de ser mujer" agrega algo significativo, elucida el problema y lo hace práctico (moral y normativamente)? Parecería que más bien empantana la discusión y es producto de arduas y no siempre fructíferas discusiones teóricas.

A mi juicio, los problemas del concepto serían: 1) tal como está planteada la definición, la condición necesaria de "por el hecho de ser mujer" es asemántica fuera de la idea de develación de las estructuras de poder de hombres sobre mujeres e ilógica como proposición; 2) es ilógica como proposición porque no existe posibilidad de que un hombre mate a una mujer ni por el hecho de serlo ni por el hecho de no serlo; 3) la proposición de Russell concibe la relación hombre/mujer como binarismo en un universo con solo dos subconjuntos posibles, lo que además puede llevar una tendencia de asumir la idea esencialista y metafísica de que un grupo es víctima y el otro victimario; 4) las preguntas complementarias que se presentan en este trabajo evidencian lo oscuro o abstruso del concepto; 5) no puede ser comprendido claramente como un delito de odio, pues la condición de group bias es difícil de determinar o inexistente; 6) por lo general, no puede ser tomado como un delito de odio, porque no hay ningún metamensaje detrás, pues los delitos son dirigidos contra personas en concreto; sin embargo, existen casos en los que sí puede existir este componente; ${ }^{15}$ 7) las críticas que Russell hace al concepto de feminicidio de Lagarde pueden aplicarse de forma análoga al suyo; 8) es un concepto sin posibilidades de instrumentalidad jurídica reales.

Quisiera recalcar de forma central que ante la dificultad probatoria del móvil del asesinato - "por el hecho de ser mujer"-, se puede terminar apelando a los componentes teóricos (las causas estructurales de los asesinatos), lo

\footnotetext{
15 Agradezco a uno de los lectores anónimos de este trabajo, quien sugirió revisar este argumento con el contraejemplo de los crímenes por honor que sí tienen, además del castigo particular, una función ejemplificadora general hacia conductas similares en otras personas y por tanto sí transmiten un "metamensaje" como lo denomina Zaffaroni.
} 
que termina por ubicar a los acusados dentro de un grupo y crear sesgos que contravienen principios de justicia y debido proceso. Subrayo que el concepto puede funcionar en sentido teórico, pues connota formas sociales repetidas detrás de los actos; pero lo que tiene cabida en la teoría, ya sea estadística o analíticamente, no puede ser un elemento procesal al juzgarse casos particulares.

Justamente uno de los problemas sea que el concepto, en vez de pensarse de forma lógica y práctica, pondera demasiado los factores "teóricos" que discute o de los cuales es producto. En un mundo, en el que la mayoría de las víctimas de violencia de género son mujeres, y en el que la mayoría de perpetradores son hombres, este concepto ayuda muy poco, e incluso es nocivo en el sentido en que podría también "naturalizar" la idea de "hombre" como victimario "por el hecho de serlo", además de victimizar a la mujer. El término tiene relevancia porque visibiliza la problemática de aquellas relaciones de poder que denuncia, pero probablemente sea más sencillo partir de tales condiciones asimétricas y considerar factores agravantes para sancionar los casos particulares que se juzga. Para ello, habría que prescindir de una condición necesaria del concepto que además funciona como motivación del victimario: "por el hecho de ser mujer".

\section{Bibliografía}

Bueno, Gustavo (1999). "Predicables de identidad". El Basilisco 25, pp. 3-30.

Butler, Judith (2007). El género en disputa. El feminismo y la subversión de la identidad. Barcelona: Paidós.

Brown, Wendy (2000). “Suffering Rights as Paradoxes”. Constellations Vol. 7 N² 2, pp. 230-241.

Calderón Gordo, Sharon (2005). "Ni «género» ni «sexo»". Catoblepas 46, p. 13.

CAmpbell, J. et al. (2003). "Risk Factors for Femicide in Abusive Relationships: Results from a Multisite Case Control Study". American Journal of Public Health Vol. 93 $\mathrm{N}^{\circ}$ 7, pp. 1089-1097.

Campbell, J., Webster, D., Glass, N. (2009). "The Danger Assessment: Validation of a Lethality Risk Assessment Instrument for Intimate Partner Femicide”. Journal of Interpersonal Violence Vol. $24 \mathrm{~N}^{\circ} 4$, pp. 653-674. 
Caputi, J., Russel, D. (1990). "Femicide: Speaking the Unspokable”. Ms 1 (2), pp. 34-37.

Carcedo, A., Ordóñez, C. (2011). "Femicidio en Ecuador”. Comisión de transición hacia el Consejo de las Mujeres y la Igualdad de Género.

Chejter, Silvia (2008). Femicidios: desafíos teóricos y perfiles estadisticos. Buenos Aires: Cecym.

Corradi, Consuelo - Marcuello-Servós, Chaime - Boira, Santiago - Weil, Shalvia (2016). "Theories of femicide and their significance in social research". Current Sociology, pp. 1-21.

Fuentes Osorio, J.L. (2017). "El odio como delito". Revista Electrónica de Ciencia Penal y Criminología 19-27, pp. 1-52.

Gerstenfeld, Phylis (2017). "Hate Crimes". En: The Wiley Handbook of Violence and Agression.

Hansen, Jenniffer (2019). “Continental Feminism”. The Stanford Encyclopedia of Philosophy.

Humbert, Michel (1988). "Le tribunat de la plèbe et le tribunal du peuple: remarques sur l'histoire de la provocatio ad populum." En: Mélanges de l'École française de Rome. Antiquité, tomo 100, Nº 1 pp. 431-503.

Jаков, GüNTher (2004). "Imputation in Criminal Law and the Conditions for Norm Validity”. Buffalo Criminal Law Review, Vol. 7 No. 2, pp. 491-511.

Kant, Immanuel (1999). Prolegómenos a toda metafísica. Madrid: Itsmo.

- (2002). Crítica de la razón pura. Madrid: Tecnos.

Kelsen, Hans (1965). Teoría pura del derecho. Buenos Aires: EUDEBA.

Lagarde, Marcela (2006). "Del femicidio al feminicidio". Desde el jardín de Freud, N 6, pp. 216-225.

Laurenzo Coppello, Patricia (2012). “Apuntes sobre el feminicidio”. Revista de derecho penal y criminología 3 N 8, pp. 119-143.

McAffe, NoËlle (2018). "Feminist Philosophy". The Stanford Encyclopedia of Philosophy.

Marín de Espinosa, Elena (2017). "El marco normativo de la violencia de género: un estudio de derecho comparado acerca de las leyes de segunda generación y de la Ley Integral Española”. Revista de Derecho Penal y Crimonología 3ra época No 17 , pp. 93-126. 
Mathews, S., Abrahams, N., Jewkes, R., Lombard, C., Martin, L., Van Der Merwe, L., Vettel, L. (2009). 'Cada seis horas': femicidio íntimo en África del Sur”. En Irene Agudelo y Ruth Largaespada: Fortaleciendo la comprensión del femicidio: de la investigación a la acción. Washington: PATH.

Mendoza, Regina (2018). "Cifras de femicidios en México durante el sexenio de EPN". Cultura Colectiva. https://news.culturacolectiva.com/mexico/cifras-de-feminicidios-en-mexico-durante-sexenio-de-epn/

Mir Puig, Santiago (1994). "La imputabilidad en derecho penal". En: Centro de Estudios Judiciales. Colección Cursos Vol. 3, Jornadas de psiquiatría forense, pp. 35-50.

Peláez Mejía, José (2018). "La necesidad del análisis causal frente a la teoría de la imputación objetiva en el derecho penal". Revista de Derecho Valdivia Vol. XXXI $\mathrm{N}^{\circ} 2$, pp. 295-320.

Pierobom De Ávila, Thiago (2018). “The crimonalisation of femicide”. En: K. Fitz-Gibbon, S. Walklate, J. McCulloch, J.M. Maher (ed.) Intimate partner violence, risk and security. Securing women's lives in a global World. Londres y Nueva York: Routledge.

Pola, Susi (2009). "Feminicidio en República Dominicana”. En: Irene Agudelo y Ruth Largaespada: Fortaleciendo la comprensión del femicidio: de la investigación a la acción. Washington: PATH.

Poliakov, Lev (1969). Auschwitz. Barcelona: Orbis.

Racca, Ignacio (2015). "Análisis crítico sobre el tipo penal de femicidio". Revista de Pensamiento Penal. URL: http://www.pensamientopenal.com.ar/system/ files/2015/08/doctrina41797.pdf

Russell, D., Harmes, R. (2001). Femicide in Global Perspective. New York and London: Teachers College Press.

Russell, Diana (2012). "Defining femicide”. Introductory speech presented to United Nations Symposium on Femicide 2012.

- (2009). "Femicidio: politizando el asesinato de mujeres". En Irene Agudelo y Ruth Largaespada: Fortaleciendo la comprensión del femicidio: de la investigación a la acción. Washington: PATH.

Saccomano, Celeste (2015). "The causes of femicide in Latin America”. Students Paper 24, Institut Barcelona Estudis Internacionals.

TARufo, Michele (2008). La prueba, artículos y conferencias. Santiago de Chile: Editorial Metropolitana. 
Toledo VÁsquez, Patsilí (2009). Feminicidio. México: OACNUDH.

Tuesta, D., Mujica, J. (2015). "Problemas en la investigación procesal-penal del femicidio en Perú". Urvio, Revista Latinoamericana de Estudios de Seguridad No 17, pp. 80-95.

Valle, Catalina (2018). Atlas de género. Quito: INEC.

\section{Diarios}

"Ni una menos: ¿Por qué Zaffaroni no cree que hay femicidio en Argentina”. El Clarin. 3/06/2015. https:/www.clarin.com/sociedad/zaffaroni-cree-existe-femicidioargentina_0_ryQtPdYvmg.html

Recibido: 12/1/2020

Aceptado: 26/5/2020

Este trabajo se encuentra bajo una licencia de Creative Commons Reconocimiento-

NoComercial-SinObraDerivada 4.0 